\title{
Articulando os direitos humanos à saúde e aos benefícios do progresso científico no processo de avaliação e incorporação de medicamentos: do global ao local
}

Articulating human rights to health and the benefits of scientific progress in the assessment and incorporation of medicines: from global to local

Articular los derechos humanos a la salud y los beneficios del progreso científico en la evaluación e incorporación de medicamentos: de global a local

Miriam Ventura ${ }^{1,2}$

Deisy de Freitas Lima Ventura ${ }^{3}$

\section{Resumo}

$\mathrm{Na}$ perspectiva dos direitos humanos à saúde e aos benefícios do progresso científico, o artigo analisa o arcabouço jurídico-institucional e argumentativo da avaliação e incorporação de novas tecnologias nos sistemas de saúde, como essencial na garantia do acesso a medicamentos. Com base na revisão documental e na literatura internacional e brasileira, investigou-se o arcabouço internacional em confronto com o brasileiro, com foco na repercussão e compatibilização dos fatos técnicos e científicos (razões extrassistemáticas) com os enunciados jurídicos (razões sistemáticas) na efetivação desses direitos. Constatouse, no âmbito local e global, o predomínio de uma concepção que prioriza a eficiência econômica e sustentabilidade dos sistemas de saúde, em detrimento à visão ética e política da incorporação das inovações farmacêuticas como um direito humano, fundamentado na solidariedade, justiça social e equidade. No âmbito nacional, houve avanços na institucionalização do processo de incorporação e avaliação, com critérios e parâmetros legais semelhantes a outros países e compatíveis com as recomendações sanitárias internacionais. As fragilidades nacionais observadas foram as flexibilizações de critérios legais na prática da incorporação que elevam o ônus probatório técnico-científico dos cidadãos para o encaminhamento dos pedidos; a ampliação e priorização de elementos econômicos e orçamentários na análise; e a ausência de previsão de participação de representantes de usuários no processo avaliativo. Conclui-se que a principal tensão nas normas e práticas analisadas com os direitos humanos é a priorização de razões predominantemente econômicas, financeiras e orçamentárias, em detrimento aos benefícios potenciais comprovados para a saúde de inovações farmacêuticas. Nesse sentido, o arcabouço analisado compromete a efetivação dos direitos humanos nas políticas de atenção à saúde, com prejuízos significativos no acesso justo e igualitário às inovações terapêuticas.

\section{Palavras-chave}

Direitos Humanos. Direito à Saúde. Avaliação Tecnológica em Saúde. Legislação \& Jurisprudência.

\footnotetext{
${ }^{1}$ Fonte de financiamento: Processo n 150892/2019-5, Pós-Doutorado Júnior/CNPq.

2 Doutora em Saúde Pública, Escola Nacional de Saúde Pública Sérgio Arouca, Fiocruz, Rio de Janeiro, RJ, Brasil; Professora associada, Instituto de Estudos em Saúde Coletiva, Universidade Federal do Rio de Janeiro. https://orcid.org/0000-0001-8520-8844. E-mail: miriam.ventura@iesc.ufr..br

3 Doutora em Direito, Universidade de Paris 1, Pantheón-Sorbonne, Paris, França; Professora titular, Departamento de Saúde Ambiental, da Faculdade de Saúde Pública, Universidade de São Paulo, São Paulo, SP, Brasil. https://orcid.org/0000-0001-8237-2470. E-mail: deisy.ventura@usp.br
} 


\section{Abstract}

From the perspective of the human right to health and to access the benefits of scientific progress, this article examines the legal, institutional and argumentative structure framing the assessment of new technologies and their and incorporation into health systems, as key to guaranteeing access to medicines. The international and Brazilian frameworks were investigated and contrasted by way of a review of documentation and of the international and Brazilian literature, focusing on the repercussions of technical and scientific facts (extrasystemic reasons) on legal statements (systemic reasons), and related reconciliation, in assuring those rights. The locally and globally conceptions were found to predominate, favouring health systems' economic efficiency and sustainability to the detriment of an ethical and political approach to incorporating pharmaceutical innovations as a human right grounded in solidarity, social justice and equity. Brazil has advanced in the process of evaluating and incorporating new medicines by introducing legal criteria and parameters similar to those of other countries and compatible with international health recommendations. The fragilities observed were instances of increasingly flexible legal criteria for the practice of incorporation, which impose a heavier onus of technical and scientific proof on citizens filing applications; the introduction and ampliation of economic and budget elements into the analyses; and the lack of any provision for user representative participation in the evaluation process, which is so highly valued in other countries. It was concluded that the main tension between the norms and practices examined and human rights is that priority is given to economic, financial and budget considerations over the proven potential health benefits of pharmaceutical innovations. In that respect, the framework examined undermines efforts to assure human rights in health care policies, thus significantly impairing fair and equal access to therapeutic innovations locally and globally.

\section{Keywords}

Human Rights. Right to Health. Health Technology Assessment. Legislation \& Jurisprudence.

\section{Resumen}

Adoptando la perspectiva de los derechos humanos a la salud y de gozar de los beneficios del progreso científico, el artículo analiza el marco jurídico-institucional y argumentativo de la evaluación e incorporación de nuevas tecnologías en los sistemas de salud, como imprescindibles para garantizar el acceso a los medicamentos. Con base en la revisión documental y en la literatura internacional y brasileña, se investigó el marco internacional en comparación con el brasileño, enfocándose en la repercusión y compatibilidad de los hechos técnicos y científicos (razones extrasistemáticas) con los enunciados legales (razones sistemáticas) en la realización. de estos derechos. A nivel local y global, predominó una concepción a favor de la eficiencia económica y la sostenibilidad de los sistemas de salud, en detrimento de la visión ética y política de la incorporación de las innovaciones farmacéuticas como derecho humano, basado sobre solidaridad, justicia social y equidad. A nivel nacional, se avanzó en la institucionalización del proceso de incorporación y evaluación, con criterios y parámetros legales similares a los de otros países y compatibles con las recomendaciones internacionales de salud. Las debilidades nacionales observadas fueron la flexibilización de criterios legales en la práctica de la incorporación, lo que aumenta la carga probatoria técnico-científica de la ciudadanía para el envío de solicitudes; la ampliación y priorización de elementos económicos y presupuestarios en el análisis; y la falta de disposiciones para la participación de representantes de los usuarios en el proceso de evaluación. Se concluye que la principal tensión en las normas y prácticas analizadas con los derechos humanos es la priorización de razones predominantemente económicas, financieras y presupuestarias, en detrimento de los probables beneficios potenciales para la salud de las innovaciones farmacéuticas. En este sentido, el marco analizado compromete 
la realización de los derechos humanos en las políticas de salud, con un daño significativo al acceso justo e igualitario a las innovaciones terapéuticas.

\section{Palabras clave}

Derechos Humanos. Derecho a la Salud. Evaluación de la Tecnología Sanitarias. Legislación \& Jurisprudencia.

\section{Introdução}

As tecnologias farmacêuticas são indispensáveis a uma atenção à saúde efetiva e de qualidade. Desde os anos 90, o acesso a essas tecnologias têm sido um dos temas principais do ativismo social em saúde e direitos humanos e dos governos, evidenciando disparidades econômicas e tecnológicas, escassez e diferenças injustas e evitáveis entre países e grupos. Os países em desenvolvimento têm acesso a terapias mais antigas, enquanto as novas alternativas são economicamente inacessíveis (1).

O direito ao acesso a medicamentos está consolidado como derivativo do direito humano à saúde (2). Observa-se, no entanto, pouca discussão sobre a necessária articulação e compatibilização entre o direito à saúde (art. 12) e o de usufruir dos benefícios do progresso científico (art. 15, § 1 "b"), ambos do Pacto Internacional de Direitos Econômicos, Sociais e Culturais. Há maior ênfase na articulação do art. 12 com o art. 15, § 1 "c", que garante a proteção da propriedade intelectual privada e seus efeitos no acesso às inovações.

No plano internacional, define-se que o acesso a medicamentos deve ser baseado em evidências científicas e nas necessidades de saúde da população e indivíduos. Seus principais atributos técnico-científicos são eficácia, eficiência e segurança, com qualidade e aceitabilidade nos contextos locais. Devem estar disponíveis para prevenção, tratamentos incluindo os paliativos, garantindo-se a acessibilidade universal e equitativa, física e econômica, com base na não discriminação, informação, participação social, atenção às populações vulneráveis e responsabilização estatal. Neste sentido, o tema vincula-se também ao campo da saúde global, tendo em vista que as dinâmicas, estruturas e relações políticas desenvolvidas no plano internacional têm ampliado a vulnerabilidade às doenças e a capacidade de reação e cumprimento pelos Estados-nacionais de suas obrigações (3).

As responsabilidades jurídicas e políticas dos Estados de proverem atenção à saúde fazem deles grandes compradores de tecnologias farmacêuticas, em um mercado globalizado com práticas comerciais que têm limitado a capacidade dos governos de provisão à população. As empresas utilizam estratégias que envolvem a busca de garantias legais nas instâncias legislativas em prol de seus interesses (hard law) e a conformação de 
relações e situações de colisão ou de cooperação com os Estados e agências internacionais, utilizando-se da soft law para criar compromissos e padrões de sujeição dos Estados ao mercado (4). Tal dinâmica tem produzido um aprofundamento das iniquidades.

Nas sociedades democráticas contemporâneas, os processos deliberativos que devem definir direitos, deveres e responsabilidades requerem forte atuação do sistema político e jurídico. Diante da intensa produção de novidades tecnológicas, amplia-se tanto a preocupação com a proteção da integridade física e psíquica das pessoas frente aos potenciais benefícios e riscos para a saúde, quanto a promoção do acesso aos benefícios científicos como um aspecto da dignidade humana.

A institucionalização da Avaliação Tecnológica em Saúde (ATS) por meio de processos públicos de incorporação de tecnologias nos sistemas de saúde tem sido a alternativa recomendada pela Organização Mundial da Saúde (OMS) na definição de prioridades e racionalização do acesso baseadas em evidências científicas e necessidades de saúde, visando à sustentabilidade dos sistemas e à garantia de maior cobertura de saúde (5). As evidências sobre a importância de adotar abordagens de direitos humanos para reduzir as iniquidades na saúde (6) e a constatação de determinantes legais na saúde (7) permitem apontar uma urgência no aperfeiçoamento das normas jurídicas e políticas; dos parâmetros éticos e técnico-científicos nos processos de incorporação; e dos mecanismos adjudicatórios, para que manejem satisfatoriamente os conflitos de interesses e atendam às necessidades individuais e coletivas da saúde. A compatibilização de interesses públicos e privados em ambiente de intensa comercialização, práticas de concorrência e monopólios torna-se cada dia mais difícil, merecendo especial atenção da OMS $(8,9,10)$. Mesmo nos países desenvolvidos do Norte, as sucessivas crises financeiras e o subfinanciamento do sistema público de saúde tornam o acesso às tecnologias um desafio (11).

No Brasil, a saúde é um direito fundamental subjetivo público, respaldado por um sistema público de saúde condizente com os valores ético-jurídicos dos direitos humanos no plano constitucional. No entanto, o subfinanciamento crônico e sua agudização têm colocado em risco o próprio direito universal e o Sistema Único de Saúde (SUS) (12).

A judicialização da política de saúde em diferentes países $(13,14)$ e as discussões nas instâncias internacionais $(8,9,15-18)$ evidenciam as dificuldades e disputas de atores públicos e privados, valores e interesses na saúde pública. No Brasil, o direito ao acesso gratuito, igualitário e universal a medicamentos no SUS consolidou-se no Superior Tribunal Federal (STF) no ano de 2009 (19, 20), firmando o entendimento jurisprudencial do exame obrigatório dos elementos clínicos de cada caso; e da comprovação da inefetividade das 
alternativas terapêuticas disponíveis no SUS para o tratamento requerido, orientada pelas evidências científicas, regulamentação sanitária e políticas públicas vigentes. O entendimento do STF impulsionou a construção de uma política judiciária protagonizada pelo Conselho Nacional de Justiça (CNJ) (21), que inclui monitoramento e arranjos interinstitucionais de suporte para a melhoria da prestação jurisdicional, em conformidade com as balizas vinculantes firmadas no STF.

A resposta do Executivo Federal foi uma acelerada revisão das políticas de assistência terapêutica - buscando conferir maior inteligibilidade de sua regulamentação - e dos protocolos de atendimento no sistema de saúde. A mobilização do Executivo e Legislativo Federal para o aprimoramento da juricidade das políticas resultou na Lei $n^{\circ} 12.401 / 2011$, que acrescentou à Lei n. ${ }^{\circ}$ 8080/1990 definições substantivas em relação à assistência terapêutica e à avaliação de tecnologias em saúde, com explicitação de direitos e deveres. Entre as alterações legislativas importantes estão a obrigatoriedade de processo administrativo para a incorporação de tecnologias e a criação da Comissão Nacional de Incorporação de Tecnologia no SUS (Conitec), com a função de assessorar o Ministério da Saúde (MS), assegurando ampla legitimidade legal ao cidadão.

A reforma legal tem sido celebrada como benéfica à redução da judicialização da saúde no Brasil (22). Mesmo que tal redução ainda não tenha logrado patamares desejáveis (23), a atuação das instâncias técnico-cientificas sanitárias incumbidas pela avaliação das novas tecnologias e sua interação com as instituições judiciais são especialmente relevantes. $A$ importância do controle democrático dos órgãos técnicos pelo Judiciário é ressaltada por Dallari (24), que destaca a responsabilidade desses órgãos na definição das prioridades por meio de avaliação técnico-científica própria e especializada das inovações.

O alcance das novas premissas legais para a assistência farmacêutica segue tensionada na tríade dos repetitivos da saúde no STF, que mantém o entendimento no sentido de não exigir a prévia incorporação, pelo Ministério da Saúde/Conitec, do tratamento demandado, desde que comprovadas sua imprescindibilidade e ausência de alternativas terapêuticas no SUS para o tratamento do demandante (Tema 106 de Recurso Repetitivo STJ [REsp n 1.657.156]; Tema 500 de Repercussão Geral - STF [RE 657.718]; Tema 006 de Repercussão Geral - STF [RE 566.471], de 19/06/2020) (25).

Apesar dos avanços na institucionalização da avaliação de tecnologias em saúde em diferentes países, ainda há poucos estudos sobre as dimensões éticas, jurídicas e sociais dessas instâncias e procedimentos. A análise dessas dimensões tem revelado as potencialidades do Direito e das instâncias judiciais e administrativas, como esferas de 
interações sociais, modelos discursivos e de (res)significação e (re)construção de leis, políticas e práticas sanitárias e jurídicas.

O estudo ora apresentado decorre de pesquisa mais ampla financiada pelo Conselho Nacional de Desenvolvimento Científico e Tecnológico (CNPq) (proc. n 150892/2019-5), que, sob o prisma da literatura crítica do Direito, dos Direitos Humanos e da Saúde Global, buscou compreender o arcabouço jurídico-institucional e as repercussões dos direitos à saúde e aos benefícios do progresso científico no acesso a medicamentos, levando em consideração como se estruturam as leis, políticas e, especialmente, o processo de incorporação de tecnologias na saúde. A discussão desenvolvida apoia-se na revisão de literatura e documental do arcabouço normativo e argumentativo internacional e nacional da incorporação tecnológica nos sistemas de saúde. O objetivo do artigo é destacar os pontos de tensão e os paralelos entre o local e o global, e a compatibilização dos arcabouços analisados com os direitos humanos.

\section{Procedimentos metodológicos}

Trata-se de pesquisa jurídico-institucional que adota a abordagem da argumentação jurídica (26). Essa abordagem permite examinar as condições que se pode considerar um argumento justificado em casos práticos, e desenvolver uma discussão racional sobre as questões de Justiça. A vantagem dessa abordagem é a ênfase no processo comunicativo entre sujeitos diferentes, que devem respeitar certas regras, sem deter-se exclusivamente aos aspectos formais.

A análise argumentativa é essencialmente contextual, e os critérios de avaliação mais importantes (e problemáticos) são os que fazem referência às noções de: universalidade; coerência; adequação das consequências, moral social e moral justificada (27). O estudo focaliza a incorporação de fragmentos do raciocínio teórico - como a comprovação de evidências científicas e econômicas (razões extrassistemáticas) indispensáveis ao cumprimento dos enunciados descritivos - pelo raciocínio jurídico - essencialmente prático, direcionado a justificar ações a partir de valores existentes em determinada norma ética ou legal, sua legitimidade e validade (razões sistemáticas).

Para o mapeamento das normas jurídicas brasileiras e internacionais vigentes, foram utilizadas fontes institucionais de acesso público. O período de abrangência da pesquisa foi de janeiro de 2015 a janeiro de 2020, incluindo-se documentos e bibliografias seminais anteriores. 
No sistema internacional dos direitos humanos, analisaram-se as Resoluções da Assembleia Geral da Organização Mundial da Saúde (WHA, sigla em inglês), da OMS; os Comentários Gerais do Comitê de Monitoramento do Tratado de Direitos Sociais, Econômicos e Culturais (CESCR, sigla em inglês) e Resoluções do Conselho de Direitos Humanos (HRC, sigla em inglês) da Organização das Nações Unidas (ONU). A pesquisa resultou em um total de cinco documentos do CESCR, quatro do HRC, e seis da WHA.

No âmbito nacional, foram selecionadas a priori as principais leis brasileiras sobre os temas abordados nas normativas internacionais, como o de pesquisa em saúde; registro de medicamentos; propriedade intelectual; política de medicamentos e de assistência farmacêutica no SUS; e sobre incorporação de novas tecnologias no SUS. Foram identificados, ao todo, 19 documentos.

A revisão da literatura foi realizada na base Scopus e no Portal de Periódicos Capes, com as seguintes chaves de busca: Health technology assessment AND global health; Health technology assessment and human rights AND Law; Access to medicines AND global health; Access to medicines AND health technology assessment; Access to medicines AND human rights; Science and technology studies AND public health AND law AND ethics; Conitec. Foram selecionados estudos sobre a implementação de instâncias governamentais de avaliação tecnológica na saúde que tratassem de aspectos éticos, legais e políticos. Do total de 854 documentos, 158 foram considerados elegíveis.

A análise do material evidenciou que as preocupações centrais se referem às barreiras de acesso relativas ao mercado; ao predomínio da propriedade intelectual e pesquisa científica privada na inovação, com escassez global de determinados tratamentos de saúde; e às fragilidades dos Estados no cumprimento de suas obrigações. Dois eixos principais orientam as normativas: a) evidências científicas articuladas com as necessidades de saúde da população e indivíduos; e b) fortalecimento das instâncias regulatórias, configurando o cenário normativo e político das práticas discursivas dos diferentes atores.

\section{O modelo regulatório de incorporação de tecnologias: estrutura e organização}

A lei brasileira confere ao Executivo competência para realizar estudo técnico prévio e definir o que deve ser ou não incorporado à assistência terapêutica no SUS. Assegura a obrigatoriedade de processo administrativo para a incorporação de tecnologias no SUS e define a Conitec como instância técnica específica do MS para a avaliação de pedidos de incorporação, inclusão, exclusão e ampliação de uso, e a elaboração e alteração de protocolos clínicos e diretrizes terapêuticas. A legitimidade é ampla - qualquer cidadão, 
organização, empresa ou órgão público pode encaminhar seus pedidos. A Conitec é um órgão colegiado com 13 membros e uma secretaria executiva. A Secretaria de Ciência, Tecnologia e Insumos Estratégicos (SCTIE) tem as atribuições administrativas e deliberativas (Decreto Presidencial $n^{\circ}$ 7.646/2011).

O propósito da ATS é o de avaliar e aplicar métodos padronizados aos processos, buscando criar condições de legitimidades social e política para superar os conflitos de interesse no processo de incorporação de tecnologias em sistemas de saúde (28). Para além do propósito de alocação de recursos, destaca-se seu valor na seleção de prioridades; na identificação de medidas de prevenção e precaução sobre o uso de tecnologias na saúde, com embasamento técnico-científico; e na garantia de direitos legais. O modelo brasileiro segue as orientações e o padrão internacional da rede The International Network of Agencies for Health Technology Assessment (NAHTA) e recomendações da OMS (5), consolidados e difundidos na maioria dos sistemas de saúde.

Estudos registram que o modelo brasileiro possui mais semelhanças do que diferenças em relação aos sistemas de outros países (28-31). Lima et al (29), ao compararem os modelos de Austrália (MSAC), Canadá (CADTH), Reino Unido (NICE) e Brasil, concluem que as diferenças observadas no Brasil se referem à composição dos comitês; apresentação de recursos à decisão; avaliação do programa; e seleção e prazos para oferta da tecnologia. Quanto à composição, Austrália e Inglaterra integram grupos de consumidores e pacientes nos comitês; o Brasil, não. A possibilidade de recurso às decisões só está prevista no processo do Reino Unido e Brasil. O prazo para disponibilização da tecnologia após a decisão de incorporação está previsto legalmente no Brasil (180 dias) e na Inglaterra (90 dias), aspecto favorável à efetividade do direito à saúde. Somente a regulamentação brasileira não se refere à realização de avaliação da atuação do órgão $(29,30)$, o que compromete a desejável accountability nos sistemas democráticos.

Outro estudo (28) compara a natureza institucional e o financiamento da atividade, identificando que Brasil, México, Estados Unidos e Suécia adotam o financiamento público direto de órgão governamental. Austrália, Inglaterra e País de Gales têm modelos de autoridade independente financiada pelo governo. Colômbia adota um modelo públicoprivado e Alemanha, privado. No Brasil, há discussão crescente sobre a transformação dessa atividade no modelo de agência, que ganha força (30).

O Canadá tem comitês provinciais/regionais e nacional, diferenciando-se de Austrália, Reino Unido e Brasil, que possuem um sistema nacional (29). Outro estudo (31) compara Brasil e Canadá, apontando como fragilidades comuns os processos e planejamentos 
regionais incipientes no campo da ATS; e como fragilidades brasileiras, a insuficiência de recursos, o impacto das decisões judiciais e a forte dependência de tecnologias provenientes do exterior.

O sistema legal brasileiro restringe a avaliação e seleção de tecnologias pelos entes federativos sem prévia e justificada necessidade de saúde pública local, autorizando o órgão federal a adotar, justificadamente, regras diferenciadas das especificidades locais (art. 28, $\S 1^{\circ}$ e $\S 2^{\circ}$, Decreto Presidencial $\left.n^{\circ} 7.508 / 2011\right)$. A organização político-administrativa do SUS - com direção única e descentralização dos serviços, acesso universal e igualitário de abrangência nacional - obriga ao tratamento isonômico dos usuários no SUS. A responsabilidade solidária e as competências sanitárias comuns dos entes federativos frente às desigualdades regionais trazem desafios para o sistema nacional, como as dificuldades no momento da pactuação das obrigações, ao mesmo tempo que ampliam a importância de planejamento e processos locais de avaliação tecnológica e maior proatividade dos gestores locais junto à Conitec.

O perfil predominante de gestores da administração pública de saúde e a não participação direta de organização da sociedade civil de usuários no sistema brasileiro têm sido objeto de críticas, considerando a recomendação internacional de imprescindibilidade do envolvimento das partes interessadas nos processos de incorporação de tecnologias (29, 30, 32). A Conitec inclui um representante do Conselho Nacional de Saúde e outro do Conselho Federal de Medicina; os demais são representantes de órgãos da administração direta e agências reguladoras (art. 19-Q, $\S 1^{\circ}$, Lei $n^{\circ}$ 8.080/90). A Conitec tem buscado ampliar os canais de comunicação da sociedade com a produção de materiais, como o Guia de Envolvimento ATS; relatórios, para sociedade, das recomendações em consulta pública; e enquetes e chamadas para registrar a experiência de associações de pacientes.

A decisão de incorporação ou não de determinada tecnologia no SUS afeta diretamente o direito dos cidadãos, especialmente, a população mais vulnerável e hipossuficiente, dependente da assistência farmacêutica pública e sem renda para aquisição direta de medicamentos. Nesse sentido as iniciativas de ampliação dos canais de comunicação com a sociedade pela Conitec, mesmo que louváveis, são insuficientes para sanar o déficit de transparência e participação social plena no processo de incorporação em conformidade com a tendência internacional. $E$ ainda, infringem a exigência legal de ampla participação da comunidade na gestão do SUS (art. 7, VIII, Lei $n^{\circ} 8.080 / 90$; e Lei $n^{\circ}$ 8.142/1990) e fragilizam a legitimidade social do processo. 


\section{O processo de incorporação de tecnologias: critérios legais e práticas de avaliação}

Destacam-se os seguintes aspectos positivos do processo de incorporação de tecnologias brasileiro: é obrigatório a deliberação por processo administrativo com regras legais procedimentais e substantivas próprias preestabelecidas; a lei garante a legitimidade ativa de qualquer cidadão ou pessoa jurídica para apresentar pedidos; há obrigatoriedade de realização de consulta pública, permitindo contribuições livres que devem ser consideradas na recomendação final; prevê audiência pública opcional, antes da tomada de decisão, a ser apreciada pelo secretário da SCTIE, responsável pela decisão.

Uma fragilidade central do modelo brasileiro fica por conta dos requisitos mínimos estabelecidos para a propositura do processo, como estudos das evidências científicas da tecnologia requerida e comparada se houver outra semelhante disponibilizada no SUS (revisão sistemática ou parecer técnico-científico); de avaliação econômica na perspectiva do SUS e de impacto orçamentário.

A atribuição do ônus probatório técnico-científico - de aspectos e na forma destacados acima - ao requerente restringe a maioria dos cidadãos e organizações, e pode, inclusive, estimular a judicialização da demanda à vista da jurisprudência, que condiciona à análise do pedido a laudo médico que comprove a imprescindibilidade do medicamento e a inexistência de alternativas no SUS para o tratamento. Também pode estimular que pacientes busquem as próprias indústrias para obterem os estudos necessários para instrução do processo administrativo, comprometendo a imparcialidade das análises.

Os desafios da institucionalização desses processos se ampliam considerando a imparcialidade; a transparência; e a complexidade da deliberação que envolve razões extrassistemáticas - provas de evidências científicas e outros elementos técnico-científicos - e razões sistemáticas do Direito, que envolve o valor social e econômico das inovações terapêuticas, como bens públicos garantidores da dignidade humana.

Novaes \& Soárez (28) apresentam o interessante modelo de Suécia, Inglaterra, País de Gales, Estados Unidos e Alemanha, que distinguem assessment e appraisal, considerando diferentes dimensões e objetivos de análise que, ao final, devem ser ponderados e deliberados por outra autoridade constituída. O assessment trata do processo técnico/científico de coleta e síntese de informações relevantes da tecnologia e é realizado por pesquisadores. O Appraisal do relatório técnico, é definido como o processo político de tomada de decisão pelos formuladores de política. É realizado por outro órgão, que leva em consideração, além do assessment, outros fatores e valores. Uma terceira parte é responsável pela decisão de incorporação e cobertura, propriamente. 
A Conitec é responsável pela avaliação (assessment) e apreciação (appraisal), e o MS, pela decisão atribuída à SCTIE. Caetano et al (12), no estudo realizado entre 2012 e junho de 2016, apontam que todas as recomendações finais da Conitec, sem exceção, foram acatadas e homologadas pelo secretário da SCTIE, sem apreciação (appraisal) de outros fatores e valores políticos, social e de direitos dos administrados. A ausência absoluta de recursos às decisões pelos proponentes é outro aspecto que chama atenção em razão do conformismo dos participantes, na sua maioria, órgãos públicos e empresas de saúde. Novaes \& Soárez (28) consideram a importância de explicitar outros critérios para além dos técnico-científicos que devem ser considerados na tomada de decisão, destacando que a Suécia é o único país que inclui implicações éticas, legais e psicossociais como critério de priorização formal para a seleção de tecnologias. A ausência de considerações éticas e relativas aos direitos dos usuários se aplica ao contexto nacional e também a outros países.

A priorização dos direitos dos usuários no processo de seleção, enquanto critério específico, é pouco explorada nos estudos consultados. O principal foco é a tecnologia avaliada, a indicação terapêutica com base na doença, e a econômica. No modelo brasileiro, a experiência das pessoas é considerada subsidiariamente no âmbito das consultas públicas, quando consideradas relevantes pelo Plenário. A não consideração de especificidades é tensionada judicialmente, na medida em que restringe tratamentos por vezes imprescindíveis. Charvel et al (30) referem-se à imprecisão dos comandos legais do art. 7, III, da Lei $\mathrm{n}^{0}$ 8.080/1990, que confere autonomia aos usuários no âmbito dos seus tratamentos, à vista do art. 19-M, que limita a assistência ao rol de procedimentos e medicamentos previstos na regulamentação do SUS. O autor (30) argumenta no sentido de afastar-se a incidência do art. 19-M, de natureza programática, diante da violação de direito fundamental previsto no referido art. 7, III, seguindo o entendimento da jurisprudência brasileira.

Os critérios legais são centrais para a garantia dos direitos dos cidadãos. A discussão jurídica-acadêmica (33) expressa preocupação com as flexibilizações e alterações realizadas no modelo legal no processo de incorporação pelos gestores. No Brasil, os gestores federais têm promovido alterações significativas nos critérios legais técnicos estabelecidos - por exemplo, a inclusão do critério de custo-efetividade e do melhor conhecimento técnico-científico disponível, introduzido pelo Decreto Presidencial $\mathrm{n}^{\circ}$ 7.646/2011, Portaria Ministerial n² 2009/2012 e Portaria da SCTIE/MS n² 26, de 12/06/2015, para submissão de proposta, ampliando o rol do art. 19-Q, §2 I e II, da Lei n 8.080/1990.

A dinâmica de ampliação e redefinição sutil de requisitos legais (hard law) pelas regulamentações e prática da avaliação (soft law) encontra paralelo em outros modelos 
regulatórios, como apontado no estudo de Bernier et al (33) sobre o modelo francês, do Reino Unido e do Canadá. As autoras apontam que práticas semelhantes na produção legislativa (hard law) - que estabelecem o que denominam de governança legal e legitimam as agências - e a soft law, com base nas práticas dos profissionais de avaliação tecnológica, vão circunscrevendo o papel social da avaliação para além de suas leis constitutivas. Tal dinâmica torna esses atores sociais responsáveis por definir, estruturar e operacionalizar a implementação do processo na prática, dando novos contornos ao papel estrutural e social do processo de avaliação. Concluem que, apesar da existência legal e de estruturas predeterminadas, a legitimidade normativa da avaliação tecnológica não é totalmente estabelecida pela hard law, pois depende da soft law. A principal crítica é uma persistente imprecisão na governança jurídica.

A linguagem utilizada nos Relatórios da Conitec, apesar da rica descrição dos métodos e dados, não permite identificar facilmente a coerência e adequação da decisão; a ponderação entre os fatos comprovados e suas consequências; entre a priorização de determinados elementos e não outros. O tratamento isonômico, fundamentado no princípio da igualdade, por exemplo, é um dos mais comuns anseios de justiça identificados nas sociedades. Assim, os Relatórios de Recomendação e as Deliberações devem ser claros, objetivos, e explicitar os critérios e pesos adotados. A comunicação entre o interlocutor e o destinatário direto, ou todos que tomem conhecimento dela, deve ser plena. Nesse particular, a linguagem e a argumentação são de fundamental importância.

As inconsistências argumentativas - imprecisão e/ou inadequação de alguns relatórios - observadas no estudo são corroboradas por estudos de especialistas da Saúde Coletiva $(12,28,29,31,32)$ e do Direito $(34,35)$, e serão adiante apontadas, enfatizando-se os elementos relativos às evidências científicas e econômicas. No caso brasileiro, as imprecisões sugerem que os agentes públicos não se atentaram para a natureza diferenciada dos atos administrativos próprios do procedimento administrativo e seus requisitos de validade. A jurisdição administrativa não tem sido exercida de forma eficiente e adequada à expectativa da sociedade e especialistas.

\section{Evidências científicas e econômicas: de que tipos de evidências precisamos para promover saúde e direitos?}

O direito humano de usufruir dos benefícios do progresso científicos exige que esses benefícios sejam efetivos e seguros; que as intervenções em saúde sejam baseadas nas melhores evidências científicas; e que esse conhecimento esteja disponível para 
formuladores de políticas, profissionais, usuários, cuidadores e todos os tomadores de decisão em saúde (36). A importância da comprovação das evidências científicas se assenta na obrigação moral e legal de agir e de promover benefícios às pessoas que, na assistência à saúde, parece clara e inconteste. Sua força exortatória faz crer que seu cumprimento é simples, e que o exame das evidências científicas possa aferir um valor certo e absoluto das tecnologias na saúde.

Todavia, avaliar os benefícios de algo ou de uma ação não é simples. Depende de uma valoração específica sobre o bem a ser protegido ou alcançado, sempre muito difícil nas sociedades pluralistas. Exige, ainda, que se estabeleça o conteúdo da ação previamente, o que também nem sempre é possível, considerando-se a complexidade e a diversidade dos contextos em que se dão os conflitos e as necessidades de saúde. O uso das tecnologias em saúde ganha contornos mais complexos e envolve outros valores no cuidado em saúde, que são construídos intersubjetivamente para alcançar um êxito técnico e um sucesso prático (37). Neste sentido, podem-se definir evidências científicas como a "combinação da melhor evidência de pesquisa, da experiência do profissional de saúde e dos valores do paciente" (36). Mas a definição de evidências científicas na saúde não é uníssona e conta com discussões acerca da validade dos métodos utilizados e da generalização de suas afirmações, que "reflete uma disputa discursiva por legitimidade científica e política sobre questões de saúde" (36). No mesmo sentido, Camargo Jr. (38) afirma que uma sólida base de conhecimentos científicos é uma das condições necessárias para garantir a saúde como um direito humano, porém, o empreendimento científico pode ser igualmente fonte de violações de direitos humanos. Recentemente, com ataques de múltiplas fontes que tentam minar o conhecimento científico perfeitamente razoável, há que se manter uma postura crítica compatível com a defesa do conhecimento científico adequado que, de fato, colabore sem cair na armadilha do cientificismo (38).

Se as evidências, ao mesmo tempo científicas e políticas, contam com um tipo de argumentação própria na saúde, que deve ser cuidadosamente desenvolvida, na vigência dos direitos humanos nos Estados de direito democráticos, essa argumentação deve ser compatibilizada com a argumentação jurídica orientada pelos valores ético-jurídicos e de justiça social que fundamentam o sistema normativo. A questão não é retórica, tampouco meramente teórica; seu alcance prático é imenso e está posto na discussão da judicialização da saúde. A dificuldade é apontada pelo jurista Ciarlini (34), quando questiona: "o que terá restado ao trabalho jurisdicional para avaliar adequadamente a possibilidade de defender a existência de uma situação jurídica designativa do direito à saúde, em face do Estado?” (33). 
A posição elevada que as evidências científicas ocupam nas práticas de saúde deve ser compreendida em sua complexidade, imprescindibilidade e hierarquia no processo decisório. A eficácia, efetividade e segurança de uma tecnologia em relação aos seus benefícios clínicos, riscos e efeitos adversos, são consensualmente admitidas como os atributos mais importantes para sua incorporação. No Brasil, o exame desses três atributos é obrigatório. A comprovação da segurança e da eficácia é também requisito obrigatório para o registro do medicamento pela Agência Nacional de Vigilância Sanitária (Anvisa). A efetividade é uma dimensão mais complexa de avaliação, por considerar os benefícios da tecnologia para uma população específica em condições normais de uso, dependente de dados clínicos relativos ao uso (30). A lei brasileira veda a incorporação de medicamentos não registrados na Anvisa, mas estudo das recomendações da Conitec no período de 20122016 identifica que seis dos 93 medicamentos incorporados ao SUS no período não possuíam registro na Anvisa (40). Considerando as possíveis exceções legais em prol da saúde pública, o dado merece ser examinado em perspectiva ético-jurídica mais densa.

Os estudos usualmente utilizados para sintetizar as evidências científicas da tecnologia são o ensaio clínico controlado randomizado (ECR) e não randomizado, estudos clínicos sem seleção aleatória de pacientes e estudo de coorte. Os métodos para a realização das sínteses são a metanálise, técnica que utiliza métodos estatísticos para revisar e interpretar criticamente os resultados e obter sínteses quantitativas sobre os efeitos das tecnologias; e a revisão sistemática, que a partir de uma pergunta claramente formulada usa métodos sistemáticos e explícitos para identificar, selecionar e avaliar criticamente pesquisas relevantes, além de coletar e analisar dados diversos dos estudos incluídos na revisão (39). A pergunta do estudo avaliativo deve incluir a indicação pretendida e se refletirá em todo o processo de análise, além de indicar qual o método mais adequado na busca da evidência.

Considerando as disputas discursivas sobre quais evidências seriam mais válidas para indicar a incorporação de determinada tecnologia para um tratamento específico, bem como o grau de incerteza e imprecisão comum, busca-se estabelecer uma hierarquia de evidência, que pode ser definida como a capacidade de cada estudo minimizar vieses. Como aponta Diniz (36), os esforços de minimização de vieses são centrais à qualidade da avaliação, e devem ser consideradas outras dimensões influenciadoras, como o relato do desenho da pesquisa e sua inclusão no registro obrigatório dos ensaios clínicos, as fontes de financiamento e os potenciais conflitos de interesses.

A avaliação econômica comparativa dos benefícios e dos custos em relação às tecnologias a serem incorporadas é outra premissa legal obrigatória no processo de 
incorporação (art.19-Q, § $2^{\circ}$ II, Lei n 8.080/1990). As variáveis previamente estabelecidas na regulamentação da lei pelo gestor (39) são: i) custo em saúde: valor dos recursos empregados no uso de uma alternativa terapêutica, de um programa ou de um serviço de saúde, durante um período de tempo; ii) custo-efetividade: as consequências (resultados) são medidas em unidades naturais (p.ex. anos de vida ganhos ou eventos clínicos evitados); iii) custo unitário: valor pago por unidade da tecnologia; iv) custo-utilidade: as consequências (resultados) das tecnologias em saúde são mensuradas como preferências (p.ex. anos de vida ajustados por qualidade); e v) impacto orçamentário: consequências financeiras em determinado cenário de saúde com recursos finitos.

A regulamentação brasileira não exige uma avaliação econômica completa, mas o Decreto $n^{\circ} 7.646 / 2011$ (art. 3, item 4) e a Portaria GM/MS n² 2009/2012 estabelecem como essencial o custo-efetividade para o sistema e para o cidadão. Na discussão sobre a judicialização da saúde, a análise econômica do Direito tem ganhado espaço de destaque e apoio de juristas que, entre outras questões relevantes, argumentam a imprescindibilidade do custo-efetividade como medida decisiva na incorporação de medicamentos, sustentando que o direito à saúde não é absoluto, que todo tratamento tem custo e que os recursos financeiros são limitados, devendo-se estabelecer limiares, especialmente à vista do teto de gastos prescrito na Emenda Constitucional n. ${ }^{\circ} 45(41,42)$.

O conceito de limiar de custo-efetividade é uma razão entre um custo monetário e uma medida de ganho em saúde no denominador. A adoção de um limiar para determinado sistema de saúde pode ser explícita ou implícita. No Brasil, não há um limiar explícito, como no Canadá e na Austrália. Há uma iniciativa do Senado brasileiro (PL n 415/2015) para incorporá-lo, prevendo divulgação do parâmetro utilizado na análise das solicitações de incorporação de tecnologias no âmbito do SUS. Argumenta-se que o vácuo legal traz insegurança jurídica em razão da imprecisão nos relatórios da Conitec. A pertinência de se estabelecer legalmente um limiar para o custo-efetividade no Brasil segue sem consenso $(43,44)$.

A avaliação econômica não tem sido decisiva nas deliberações da Conitec, e quando incluída, não é adequadamente desenvolvida para atender ao objetivo de alocação de recursos em saúde. Estudo que analisou os elementos da decisão de incorporação (12) apontou que apenas $14 \%$ referem-se às evidências de custo-efetividade em relação às opções disponíveis; também observou a hierarquia superior e prevalente das evidências clínicas, com 40,9\% dos processos com justificativa relacionada ao "benefício clínico adicional", seguido da "necessidade clínica não preenchida" (21,5\%). O maior percentual, 
abaixo da justificativa de "benefício clínico adicional", ficou por conta do baixo impacto financeiro-orçamentário (25,8\%) previsto nas regulamentações (12).

As variações de preço registradas no custo em saúde entre os preços apesentados pelos demandantes e o referente ao preço máximo de venda ao governo (PMVG) e os preços com descontos é outro elemento observado nas análises. A preocupação nacional encontra paralelo na discussão internacional, com a recomendação e medidas da OMS para a criação de um banco de preços internacionais que favoreça a transparência e negociações dos países, considerando as discrepâncias identificadas entre países e de preços praticados mesmo internamente (9).

A tensão em torno da inclusão da avaliação econômica na lei federal foi objeto de veto presidencial afastado pelo Legislativo, que manteve a previsão. As regulamentações do gestor federal ampliaram as exigências, incorporando a obrigatoriedade do impacto da incorporação da tecnologia no SUS, e outros elementos referentes à relevância da incorporação para as políticas prioritárias do SUS, as condicionantes relativas ao preço máximo para incorporação e critérios técnico-assistenciais para alocação, quando pertinentes.

\section{Considerações finais}

As necessárias considerações éticas e jurídicas dos direitos humanos à saúde e o de usufruir dos benefícios do progresso científico, enquanto valores que devem orientar a ação pública, são ausentes na maior parte dos modelos regulatórios de incorporação de tecnologias utilizados no mundo. Os processos públicos de incorporação de tecnologias em saúde constituem campo próspero para se pensar como avançar na realização desses direitos, por sinalizar outras dimensões e valores em jogo implicados no cumprimento das obrigações estatais, e alternativas na superação das barreiras de acesso. Como assevera Donders (45), o direito de usufruir dos benefícios científicos no domínio da saúde recebeu pouca atenção dos Estados, órgãos da ONU e programas acadêmicos. Apesar de o papel da ciência nas sociedades e seus benefícios e perigos potenciais estarem presentes em vários fóruns internacionais, quase nunca estão em um contexto de direitos humanos.

Constata-se, na análise das discussões sobre os modelos e práticas regulatórias de incorporação de novas tecnologias nos sistemas de saúde, que o cumprimento de um dever tão objetivo como a comprovação das evidências científicas e dos benefícios de determinada tecnologia é realizada de forma abrangente, heterogênea e por vezes imprecisa, permitindo uma relativização do valor das evidências dos benefícios na definição do conteúdo dos direitos humanos implicados. Essa relativização sugere uma captura da avaliação da 
tecnologia pela lógica do racionamento de recursos em prol da sustentabilidade do sistema. Diante das dificuldades econômicas e orçamentárias, é produzido um racionamento de direitos, em vez da racionalização necessária à efetivação e compatibilização dos direitos nas políticas de atenção efetivas e necessárias às pessoas.

A ótica da eficiência econômica para basear as decisões relacionadas a direitos é um critério problemático e que, em termos gerais, não pode ser considerado decisivo, pois o Direito possui outros objetivos finalísticos assentados em razões morais e sociais que se deve considerar, entendendo-se que não devem prevalecer as razões econômicas sobre as razões de correção, a depender do direito implicado. Como ressalta Atienza (27), as razões econômicas não servem igualmente a todos os ramos do Direito: se no Direito Tributário tem papel central, no Direito Penal, por exemplo, só poderia exercer uma função residual. No direito à saúde, a dimensão econômica deve também ter uma função residual, e o potencial dos benefícios do progresso científico na saúde, o critério de correção.

Para tanto, é necessário incorporar, no processo de avaliação e incorporação tecnológica na saúde, a perspectiva das pessoas e da garantia de seus direitos, com dimensões éticas, jurídicas e sociais indispensáveis à avaliação, considerando-se a exigibilidade da realização dos direitos humanos pelos governos, enfatizando-se o gozo dos benefícios científicos e limitações à propriedade intelectual privada como central na realização do direito à saúde.

A resposta do sistema internacional de Direitos Humanos, enquanto instituição voltada à mediação dos conflitos baseado nos ideais ético-jurídicos estabelecidos, demonstra fragilidades em sua efetivação, mormente em conjuntura de intensa globalização econômica e do neoliberalismo nas políticas de saúde, que promove uma erosão contínua dos direitos. Há um claro avanço da lógica mercado-economia na saúde e de uma cidadaniaconsumidora, reforçadas nos modelos regulatórios que privilegiam aspectos técnicocientíficos-burocráticos sem resguardar, com a mesma intensidade, uma governança em saúde pautada na justiça social e nos direitos humanos.

O aperfeiçoamento da jurisdição administrativa é central para a legitimidade social e legal, cujo alcance é dependente da aproximação dessas decisões administrativas com os anseios de justiça da sociedade. Nesse sentido, a adoção de uma perspectiva comunicativa e participativa voltada ao entendimento, que favoreça a (re)formulação de direitos e o redirecionamento das políticas de saúde centradas nas pessoas se faz urgente. 


\section{Referências}

1. Biehl J. Theorizing global health. Medicine Anthropology Theory. 2016; 3(2). doi.org/10.17157/mat.3.2.434

2. Hunt $P$, Khosla R. Acesso a medicamentos como um direito humano. Sur. Revista Internacional de Direitos Humanos. 2008; 5(8):100-121

3. Ventura D, Nunes J. Apresentação. Lua Nova: Revista de Cultura e Política. 2016; (98):7-16. doi.org/10.1590/0102-6445007-013/98

4. Ventura D. Direito e saúde global: o caso da pandemia de gripe $A(H 1 N 1)$. São Paulo: Outras Expressões; Dobra Editorial; 2013.

5. OMS. 2014; WHA 67.23. Health intervention and technology assessment in support of universal health coverage.

6. Yamin AE, Cantor R. Between insurrectional discourse and operational guidance: challenges and dilemmas in implementing human rights-based approaches to health. Journal of Human Rights Practice. 2014; 6(3):451-85.

7. Gostin LO et al. The legal determinants of health: harnessing the power of law for global health and sustainable development. Lancet. 2019; 393(10183):1857-1910.

doi:10.1016/S0140-6736(19)30233-8.

8. World Health Organization. WHA 68.18. Global strategy and plan of action on public health, innovation and intellectual property (GSPOA). Geneva: WHO; 2015.

9. World Health Organization. WHA 72.8. Improving the transparency of markets for medicines, vaccines, and other health products. Geneva: WHO; 2019.

10. World Health Organization. WHA 69.20. Promoting innovation and access to quality, safe, efficacious and affordable medicines for children. Geneva: WHO; 2016.

11. Faria PL, Cordeiro JV. Managing a difficult ethical and legal equilibrium in healthcare: assuring access to the basics while keeping up with innovation. Rev. Portuguesa de Saúde Pública. 2014; 3 2(2):121-122. doi: http://dx.doi.org/10.1016/j.rpsp.2014.11.001.

12. Caetano R et al. Incorporação de novos medicamentos pela Comissão Nacional de Incorporação de Tecnologias do SUS, 2012 a junho de 2016. Ciência \& Saúde Coletiva. 2017; 22(8), 2513-2525. doi: https://doi.org/10.1590/1413-81232017228.02002017.

13. Biehl J, Socal MP, Gauri V, Diniz D, Medeiros M, Rondon G, Amon JJ. Judicialization 2.0: Understanding right-to-health litigation in real time. Glob Public Health. 2019 Feb;14(2):190-199. doi: 10.1080/17441692.2018.1474483. Epub 2018 May 21. PMID: 29781395.

14. Vargas-Pelaez CM, Rover MRM, Soares L, Blatt CR, Mantel-Teeuwisse AK, Rossi FA, Restrepo LG, Latorre MC, López JJ, Bürgin MT, Silva C, Leite SN, Farias MR. Judicialization of access to medicines in four Latin American countries: a comparative 
qualitative analysis. Int J Equity Health. 2019 Jun 3; 18(1):68. doi: 10.1186/s12939-0190960-z. PMID: 31154999; PMCID: PMC6545681.

15. United Nations. CESCR General Comment n. 14 E/C.12/2000/4. The right to the highest attainable standard of health. New York: UN; 2000.

16. United Nations. WHA 67.22. Access to essential medicines. New York: UN; 2014.

17. United Nations. CESCR General Comment n. 25 E/C.12/GC/25. On science and economic, social and cultural rights. Article 15 (1) (b), (2), (3) and (4) of the International Covenant on Economic, Social and Cultural Rights. New York: UN; 2020.

18. United Nations. HRC. A/HRC/RES/23/14. Access to medicines (general resolution). New York: UN; 2013.

19. Brasil. Supremo Tribunal Federal. Saúde. Notas taquigráficas da Audiência Pública. Relator: Ministro Gilmar Mendes. Brasília; s/d.

20. Brasil. Supremo Tribunal Federal. Voto do ministro Gilmar Mendes na STA 175 AgR/CE. Brasília; s/d.

21. Conselho Nacional de Justiça. Recomendação n. ${ }^{\circ} 31$, de 30 de março de 2010. Recomenda aos Tribunais a adoção de medidas visando a melhor subsidiar os magistrados e demais operadores do direito, para assegurar maior eficiência na solução das demandas judiciais envolvendo a assistência à saúde. DJE/CNJ n 61/2010; 2010 abr. 07. p. 4-6.

22. Bucci MPD. Contribuição para a redução da judicialização da saúde: uma estratégia jurídico-institucional baseada na abordagem de direito e políticas públicas. In: Bucci MPD, Duarte CS (Coords.). Judicialização da saúde: a visão do Poder Executivo. São Paulo: Saraiva; 2017. p. 31-88.

23. Instituto de Ensino e Pesquisa (Insper), Conselho Nacional de Justiça. Judicialização da saúde no Brasil: Perfil das demandas, causas e propostas de solução. Justiça Pesquisa. São Paulo: INSPER; 2019.

24. Dallari SG. Controle judicial da política de assistência farmacêutica: direito, ciência e técnica. Physis. 2010; 20(1):57-75.

25. Pereira VRT. Tríade dos repetitivos de saúde: a judicialização após vereditos de STJ e STF. Revista Consultor Jurídico. [internet]. 2020 jul. 24 [citado em 15 out. 2021]. Disponível em: https://www.conjur.com.br/2020-jul-24/viviane-pereira-triade-repetitivossaude?imprimir=1

26. Atienza M. As razões do Direito: teorias da argumentação jurídica: Perelman, Viehweg, Alexy, MacCormick e outros. São Paulo: Ed. Landy; 2006.

27. Atienza M. Curso de argumentação jurídica. Curitiba: Alteridade; 2017. 254 p. (Coleção Direito, Retórica e Argumentação, v. 1). 
28. Novaes HMD, Soárez PC. Organizações de avaliação de tecnologias em saúde (ATS): dimensões do arcabouço institucional e político. Cadernos de Saúde Pública. 2016 nov 03; 32(Supl. 2), e00022315. Epub 2016 nov 03. Disponível em: https://doi.org/10.1590/0102$311 \times 00022315$

29. Lima SGG et al. O processo de incorporação de tecnologias em saúde no Brasil em uma perspectiva internacional. Ciência \& Saúde Coletiva. 2019 May 30; 24(5):1709-1722. Disponível em: https://doi.org/10.1590/1413-81232018245.17582017

30. Charvel S, Cobo F, Larrea S, Baglietto J. Challenges in priority setting from a legal perspective in Brazil, Costa Rica, Chile, and Mexico. Health and Hum. Rights. 2018; 20(1):173-84.

31. Silva HP, Elias FTS. Incorporação de tecnologias nos sistemas de saúde do Canadá e do Brasil: perspectivas para avanços nos processos de avaliação. Cadernos de Saúde Pública, 35 (Suppl. 2), e00071518. Epub Aug. 15, 2019. Disponível em:

https://doi.org/10.1590/0102-311x00071518

32. Aith FMA et al. Os princípios da universalidade e integralidade do SUS sob a perspectiva da política de doenças raras e da incorporação tecnológica. Revista de Direito Sanitário. 2014; 15(1):10-39. [citado em 23 set. 2016]. Disponível em: https://bit.ly/2U345IM.

33. Bernier L et al. Legal governance in HTA: environment, health and safety issues. Ethical, legal and social issues (EHSI/ELSI), the ongoing debate. Canadian Journal of Bioethics. 2020; 3(1).

34. Ciarlini ALA. Análise jurídica dos critérios axiológicos de avaliação de medicamentos pela Comissão Nacional de Incorporação de Tecnologias ao SUS - Conitec. Cad. Ibero Am. Direito Sanit. 2016; 5(1):205-219.

35. Morozowski AC. A imprescindibilidade da transparência e da adequada fundamentação no processo de incorporação de tecnologias no SUS. Migalhas. $2019 \mathrm{~b}$ [citado 25 out. 2019]. Disponível em: https://www.migalhas.com.br/depeso/313653/a-imprescindibilidadeda-transparencia-e-da-adequada-fundamentacao-no-processo-de-incorporacao-detecnologias-no-sus

36. Diniz S. Evidência em saúde: o que são, o que podem ser, e de que tipo de evidências precisamos. In: Paiva V, França Jr I, Kalichman AO (orgs.). Vulnerabilidade e direitos humanos: prevenção e promoção da saúde. São Paulo: Juruá; 2012. p. 49-50.

37. Ayres JRCM. Uma concepção hermenêutica de saúde. Physis. 2007; 17(1):43-62.

38. Camargo KR. Science, health, and human rights. Global Public Health. 2021. [citado em 15 out. 2021]. Disponível em: https://doi.org/10.1080/17441692.2021.1950800

39. Brasil. Ministério da Saúde. Secretaria de Ciência, Tecnologia e Insumos Estratégicos. Departamento de Ciência e Tecnologia. Diretrizes metodológicas: elaboração de pareceres técnico-científicos / Ministério da Saúde, Secretaria de Ciência, Tecnologia e Insumos 
Estratégicos, Departamento de Ciência e Tecnologia. 2. ed. rev. e ampl. Brasília: Ministério da Saúde; 2014. 62 p.

40. Pedro EM, Caetano R, Teodoro CRS, Steffen RE, Silva RM. Incorporação de medicamentos sem registro sanitário no SUS: um estudo das recomendações da Comissão Nacional de Incorporação de Tecnologias no período 2012-2016. Visa em Debate [Internet]. 2018 ago. 31; 6(3):12-21. Disponível em:

https://visaemdebate.incqs.fiocruz.br/index.php/visaemdebate/article/view/1042

41. Morozowski AC. A imprescindibilidade da análise mínima do custo-efetividade nas ações de saúde. Revista da Escola de Magistratura do TRF da 4. ${ }^{a}$ Região. 2019a; 12(5): 245-254.

42. Ferraz OLM, Vieira FS. Direito à saúde, recursos escassos e equidade: os riscos da interpretação judicial dominante. Dados. 2009; 52(1):223-251. doi:

https://doi.org/10.1590/S0011-52582009000100007

43. Soarez PC, Novaes HMD. Limiares de custo-efetividade e o Sistema Único de Saúde. Cad Saude Publica. 2017 maio 18; 33(4):e00040717.

44. Santos MS et al. Contradições e o limiar de custo-efetividade. Cadernos de Saúde Pública [online]. 2017; 33(8):e00096117. Disponível em: https://doi.org/10.1590/0102$311 \times 00096117$

45. Donders $Y$. The right to enjoy the benefits of scientific progress: in search of state obligations in relation to health. Med Health Care and Philos. 2011; 14(371). Disponível em: https://doi.org/10.1007/s11019-011-9327-y

\section{Colaboradores}

As autoras contribuíram com a concepção, elaboração, redação, revisão e aprovação do artigo.

\section{Como citar este artigo}

Ventura M, Ventura DFL. Articulando os direitos humanos à saúde e aos benefícios do progresso científico no processo de avaliação e incorporação de medicamentos: do global ao local. Cadernos Ibero-Americanos de Direito Sanitário. 2021 dez.;10 (Suplemento):11-31.

https://doi.org/10.17566/ciads.v10iSuplemento.856 